\title{
Sensory and Multisensory Responses in the Newborn Monkey Superior Colliculus
}

\author{
Mark T. Wallace and Barry E. Stein \\ Department of Neurobiology and Anatomy, Wake Forest University School of Medicine, Winston-Salem, \\ North Carolina 27157
}

\begin{abstract}
Superior colliculus (SC) neurons have the ability to synthesize information from different sensory modalities, resulting in enhancements (or depressions) of their activity. This physiological capacity is, in turn, closely tied to changes in overt attentive and orientation responses. The present study shows that, in contrast to more altricial species, many deep layer SC neurons in the rhesus monkey are multisensory at birth. Such neurons can respond to stimuli from different sensory modalities, and all convergence patterns seen in the adult are represented. Nevertheless, these neurons cannot yet synthesize their multisensory inputs. Rather, they respond to combinations of crossmodal stimuli much like they respond to their individual modality-specific components. This immature property of multisensory neurons is in contrast to many of the surprisingly sophisticated modality-specific response properties of these
\end{abstract}

neurons and of their modality-specific neighbors. Thus, although deep SC neurons in the newborn have longer latencies and larger receptive fields than their adult counterparts, they are already highly active and are distributed in the typical adult admixture of visual, auditory, somatosensory, and multisensory neurons. Furthermore, the receptive fields of these neurons are already ordered into well organized topographic maps, and the different receptive fields of the same multisensory neurons show a good degree of cross-modal spatial register. These data, coupled with those from cat, suggest that the capacity to synthesize multisensory information does not simply appear in SC neurons at a prescribed maturational stage but rather develops only after substantial experience with cross-modal cues.

Key words: cross-modal; multisensory integration; superior colliculus; sensory development; visual; auditory; somatosensory
A characteristic feature of many neurons in the adult superior colliculus (SC) is the ability to engage in multisensory integration, a process by which information from different sensory modalities is synthesized and transformed into an altered product (Stein and Meredith, 1993). When cross-modal stimuli are derived from the same location, as when they are initiated by the same event, they generally produce multisensory interactions that significantly enhance the response of the neuron above that elicited by the most effective of the individual modality-specific stimuli. However, when these same stimuli are derived from different locations (as when initiated by different events), they produce either no multisensory interaction or an inhibitory effect that significantly depresses the response of the neuron (Meredith and Stein, 1996; Wallace et al., 1996; Kadunce et al., 1997). These physiological changes are paralleled by similar effects on SC-mediated overt behaviors: cross-modal cues that are in spatial register significantly enhance orientation responses, and those that are spatially disparate significantly degrade these responses (Stein et al., 1989).

The nature of multisensory integration in SC neurons and the principles governing this process seem straightforward and intuitive. What is less clear, however, is whether these physiological characteristics reflect inherent properties of SC neurons that can be expressed early in life, perhaps so that they can play a role in

\footnotetext{
Received May 29, 2001; revised July 23, 2001; accepted July 27, 2001.

This work was supported by National Institutes of Health Grants NS22543 and NS36916. We thank Nancy London for her assistance in all phases of this project.

Correspondence should be addressed to Dr. Mark T. Wallace, Department of Neurobiology and Anatomy, Wake Forest University School of Medicine, WinstonSalem, NC 27157. E-mail: mwallace@wfubmc.edu.

Copyright (C) 2001 Society for Neuroscience $0270-6474 / 01 / 218886-09 \$ 15.00 / 0$
}

facilitating neonatal orientation behaviors, or whether they develop only after extensive postnatal maturation, perhaps so that they can be crafted by specific sensory experiences. Previous studies in cat have been consistent with the latter (Wallace and Stein, 1997), but the cat is an altricial species. Its sensory systems are poorly developed at birth, and the visual, auditory, and somatosensory properties of its SC neurons require extensive postnatal maturation before they can approximate the information processing capabilities they will have in the adult (Stein et al., 1973a,b, 1980; Norton, 1974; Stein and Gallagher, 1981; Kao et al., 1994; Wallace and Stein, 1997). Therefore, the delayed maturation of multisensory integration in cat SC neurons may reflect the general early immaturity of the sensory apparatus in this species, the need for extensive postnatal experience, or a combination of both factors.

The rhesus monkey, on the other hand, is comparatively mature at birth. Its visual, auditory, and somatosensory systems are already functional, and its superficial layer visual SC neurons have many properties that are surprisingly adult-like (Wallace et al., 1997). Indeed, although not yet fully mature, the sensory systems of the newborn monkey are already capable of supporting many complex behaviors (King et al., 1974; Carlson, 1984; Boothe et al., 1985; Kiorpes and Movshon, 1998). What the newborn monkey does not have, however, is extensive cross-modal experience with visual, auditory, and somatosensory cues. Consequently, the present experiments were initiated to determine whether multisensory neurons are present in this species at birth and, if so, to examine their organization and the possibility that they are already capable of integrating their cross-modal inputs.

Parts of this work have been published previously in abstract form (Wallace et al., 1995). 


\section{MATERIALS AND METHODS}

All procedures were performed in accordance with National Institutes of Health guidelines (publication number 86-23) and were conducted under an approved protocol of the Institutional Animal Care and Use Committee of the Wake Forest University School of Medicine. All data were acquired in single acute recording sessions from each of two newborn rhesus monkeys (Macaca mulatta). Recordings were begun 4-10 hr postpartum. The data generated in these experiments were then compared with an adult database in which all stimuli and data collection techniques were identical (Wallace et al., 1996).

Surgical preparation. Anesthesia was induced with ketamine hydrochloride $(10 \mathrm{mg} / \mathrm{kg}$, i.m.). Animals were intubated and maintained during surgery with gaseous anesthesia (isoflurane, 1-4\%). Body temperature was maintained at $37^{\circ} \mathrm{C}$ with a circulating hot-water heating pad. Animals were placed in a stereotaxic head holder, and a craniotomy was performed to expose the cortex overlying the SC. A hollow recording cylinder-head mount was affixed to the skull and positioned over the craniotomy (McHaffie and Stein, 1983). This device allowed the animal to be removed from the stereotaxic apparatus and supported the head without pressure points and without obstructing the eyes, pinnas, face, or body.

Recording. Anesthesia was maintained during recording with isoflurane $(0.5-1.0 \%)$. End tidal $\mathrm{CO}_{2}$ was monitored and maintained between 3.5 and $4.5 \%$. The femoral vein was cannulated for the administration of fluids (lactated Ringer's solution; $3-6 \mathrm{cc} / \mathrm{hr}$ ) and paralytic (pancuronium bromide; $2 \mathrm{mg} \cdot \mathrm{kg}^{-1} \cdot \mathrm{hr}^{-1}$ ). Animals were artificially respired, and heart rate and EEG were regularly monitored to ensure adequate depth of anesthesia. Using an ophthalmoscope, the optic discs were projected onto a translucent Plexiglas hemisphere positioned $45 \mathrm{~cm}$ from the eyes. Corrective contact lenses were applied to the anesthetized $(0.5 \%$ ophthalmic proparacaine) cornea to focus the eyes onto the screen and to prevent corneal drying.

Recording procedures. Polyamide-insulated tungsten microelectrodes ( $Z=1-3 \mathrm{M} \Omega$ at $1 \mathrm{kHz}$ ) were advanced by means of a manually driven micromanipulator to the dorsal surface of the SC. On the appearance of the multiunit visual activity characteristic of the superficial SC, the microelectrode was further advanced by means of a hydraulic microdrive. Because multisensory neurons are found in the intermediate and deep laminas, the electrode was advanced rapidly through the superficial layers, which were characterized by visual responses with small receptive fields. Once in the intermediate layers, as determined by larger visual receptive fields and activation by other sensory modalities (i.e., auditory and somatosensory), the electrode was advanced in small $(15 \mu \mathrm{m})$ steps in an effort to isolate individual neurons. Neurons were identified by their spontaneous activity and by their responses to a variety of search stimuli (described below). Neural responses were amplified, displayed on an oscilloscope, and played through an audiomonitor. Each neuron was examined for its responses to visual, auditory, and somatosensory stimuli. The initial evaluation of the modality convergence pattern and sensory responses of a neuron, as well as the manner of mapping its receptive field(s), were identical to those used in previous experiments with cat and monkey (Meredith and Stein, 1986a,b; Meredith et al., 1987; Wallace et al., 1993, 1996, 1997; Wallace and Stein, 1994).

Sensory classification, receptive field mapping, and latency testing. Visually-responsive neurons were sought using a variety of stationary and moving stimuli. Once a visually-responsive neuron was isolated, its receptive field was mapped manually using a pantoscope with which bars and spots of light could be projected directly onto the translucent hemisphere. The borders of each receptive field were determined by moving the optimum visual stimulus from the periphery inward from all directions until an enclosed responsive area was delimited. For quantitative tests of visual and multisensory responses, computer-controlled stationary and moving stimuli of a variety of shapes and sizes were used. Stimuli (luminance of $53 \mathrm{~cd} / \mathrm{m}^{2}$ against a background of $2.7 \mathrm{~cd} / \mathrm{m}^{2}$ ) were projected onto the translucent hemisphere. For moving stimuli, stimulus amplitude, direction, and speed were under independent control. An electronic shutter was used to present stationary flashed stimuli of various sizes and in various positions within and outside the excitatory receptive field.

Auditory-responsive neurons were identified by their responses to a variety of complex auditory stimuli that included hisses, clicks, claps, whistles, and broad-band $(20-20,000 \mathrm{~Hz})$ noise bursts. Auditory receptive fields were mapped using broad-band noise bursts from a pair of hoop-mounted speakers positioned $15 \mathrm{~cm}$ from each ear. Excitatory auditory receptive fields were defined on the basis of a significant difference above background activity (Meredith and Stein, 1986a,b). For quantitative tests of auditory and multisensory responses, the duration of the auditory stimuli varied from 25 to $100 \mathrm{msec}$ at intensities of $50-70 \mathrm{~dB}$ sound pressure level (SPL) (background of $\sim 50 \mathrm{~dB}$ SPL).

Somatosensory-responsive neurons were sought using taps and strokes delivered via a camel's hair brush, compression of the skin, and joint rotation. Each somatosensory receptive field was mapped using minimally effective stimuli. Somatosensory and multisensory responses were studied quantitatively using computer-controlled mechanical stimuli delivered from a probe tip mounted to a modified moving-coil vibrator. Movement of the probe tip provides no auditory cues, a fact periodically confirmed by positioning the probe tip off the skin or hair surface.

All receptive fields were plotted on standardized representations of visual, auditory, and somatosensory space (Stein and Meredith, 1993). The mean area of visual and auditory receptive fields was determined by multiplying the long and short axes of the measured receptive fields. The mean area of each somatosensory receptive field was calculated as its proportion of the cutaneous surface based on planimetric tracings. The location of each receptive field was correlated with the position of the neuron within the SC using computerized reconstruction techniques (see below). For tests of response latency, modality-specific thresholds were first determined for responses to natural physiological stimuli, and then stimuli twice this intensity were presented 10 times at $6 \mathrm{sec}$ intervals. The mean time interval to the first impulse was taken as the minimum response latency. These criteria are the same as those used in studies of adult response latencies (Wallace et al., 1996), facilitating comparisons between the two populations. ANOVA methods were used to compare response latency and vigor in the newborn and adult populations.

Multisensory tests. All quantitative tests involved the delivery of repeated, electronically controlled stimuli. Stimulus delivery and data acquisition were controlled by means of a 1401 data acquisition interface (Cambridge Electronics Design, Cambridge, UK). Once a multisensory neuron was isolated and its receptive fields were mapped, responses to each modality-specific stimulus (e.g., visual alone and auditory alone) and to the multisensory combination (e.g., visual-auditory) were quantitatively determined. The stimulus parameters (e.g., intensity, duration, etc.) were chosen based on preliminary qualitative observations and were structured to optimize the possibility of obtaining a multisensory interaction (Wallace et al., 1996; Kadunce et al., 2001). Each stimulus or stimulus combination was presented $8-12$ times at $8-15 \mathrm{sec}$ interstimulus intervals, with the different conditions interleaved. Initial analyses were structured such that the stimuli were presented within their respective receptive fields and in close spatial and temporal coincidence. In a subset of the neurons examined, the spatial and temporal relationships of the cross-modal stimuli that were presented were systematically varied so that the effects of relative spatial disparities and stimulus onset asynchronies (SOAs) could be examined.

Data acquisition and analyses. Neuronal responses to each stimulus condition (e.g., visual alone, auditory alone, and visual-auditory) were assessed based on the mean number of impulses evoked. Responses were analyzed statistically to determine whether a significant (two-tailed $t$ test; $p<0.05)$ change in the number of impulses occurred with combined stimuli compared with the most effective single-modality stimulus. The magnitude of this change was then calculated by the following formula: $\left[\left(\mathrm{CM}-\mathrm{SM}_{\max }\right) /\left(\mathrm{SM}_{\max }\right)\right] \times 100=\%$ interaction, where $\mathrm{CM}$ is the mean number of impulses evoked by the combined-modality stimulus, and $\mathrm{SM}_{\max }$ is the mean number of impulses evoked by the most effective single-modality stimulus.

Histology and death. In each electrode penetration, the depth of each isolated neuron was recorded. In addition, after a penetration, electrolytic lesions ( $10 \mu \mathrm{A}$ for $5-10 \mathrm{sec})$ were made at various depths. After the final recording penetration, the animal was killed with an overdose of sodium pentobarbital $(100 \mathrm{mg} / \mathrm{kg}$, i.v.) and perfused transcardially with saline followed by formalin. The midbrain was blocked stereotaxically, removed, and placed in sucrose overnight. Frozen sections $(50 \mu \mathrm{m}$ thickness) were taken in either the coronal or parasagittal planes and were counterstained with neutral red to facilitate visualization of laminar borders. The outline of the tissue, SC laminar boundaries, electrode penetrations, and lesions were traced using a projection microscope. These data were then entered into computer by means of a digitizing pad. Reconstruction of the tissue was performed using standard techniques so that the position of each recorded neuron could be assessed. 


\section{RESULTS}

Eleven complete electrode penetrations were made through the deep (i.e., multisensory) layers (i.e., laminas IV-V II) of the SC in two newborn animals. A total of 95 sensory-responsive neurons were isolated, and each was examined for its response characteristics. These neurons were located across the rostrocaudal and mediolateral extent of the SC. Although sensory activity was readily apparent in the SC at this developmental stage, particularly in the superficial layers (Wallace et al., 1997), the general level of activity appeared to be immature. This was especially evident in the multisensory laminas, in which background activity was weaker than in the adult and fewer multiunit sensory responses could be recorded. To evaluate the comparative incidence of sensory-responsive neurons in the multisensory laminas of the newborn and the adult (Wallace et al., 1996), every sensoryresponsive neuron with a signal-to-noise ratio of 3:1 or greater was characterized. Using this criterion, the mean incidence of isolated sensory-responsive neurons in the newborn (1.62 neurons/mm of penetration depth) was significantly lower than that of the adult $(2.27$ neurons $/ \mathrm{mm}$; $t$ test; $p<0.05)$.

In addition to this difference in incidence, sensory response latencies were found to be significantly longer in the newborn than in the adult. Because latency did not differ among modalityspecific and multisensory neurons in the two populations (see below for modality convergence patterns), the data were pooled at each age and are illustrated in Figure 1. Visual latencies showed the largest proportionate difference between the two ages, with the mean for newborns $(125.1 \mathrm{msec})$ being $52 \%$ longer than the mean for adults $(82.3 \mathrm{msec} ; p<0.01)$. Auditory latencies were $44 \%$ longer in the newborn ( 28.4 vs $19.7 \mathrm{msec} ; p<0.05)$, and somatosensory latencies were $34 \%$ longer (33.6 vs $25.0 \mathrm{msec}$; $p<0.05)$.

Typically, the vigor of the sensory responses elicited in neonates was substantially less than in adults. Again, this held true for responses to each of the effective modalities and was not different between the modality-specific and multisensory populations. Therefore, the data from these populations were pooled at each age. When presented with identical visual stimuli, the maximal response (i.e., mean number of impulses to the most effective stimulus) in the adult (averaged over 25 neurons) was nearly double (i.e., $96 \%$ greater) the maximal response in the newborn (averaged over 27 neurons; $p<0.05$ ). Similarly, the maximal response to both auditory $(73 \%)$ and somatosensory $(57 \%)$ stimuli was significantly greater in adults than in neonates $(p<0.05)$.

\section{Modality convergence patterns}

Despite this immaturity in sensory responsiveness, as in the adult, all possible modality convergence patterns were found in the SC of the newborn. The relative distribution of these neuronal types was similar at both maturational stages (Fig. 2). Nevertheless, the proportion of multisensory neurons was significantly lower in the newborn $(14.7 \%)$ when compared with the adult $\left(28.0 \% ; \chi^{2}=\right.$ 6.32 ; df $=1 ; p<0.05)$. Visual neurons were the largest component in both populations, followed by somatosensory and auditory neurons. Whereas visual-auditory neurons were the most prevalent category of multisensory neuron in the adult, visual-somatosensory neurons were the most prevalent in the newborn. In both age groups, there was the same tendency for visual and multisensory neurons (especially visually-responsive multisensory neurons) to be found in the more superficial aspects of the multisensory layers (i.e., lamina IV). In contrast, auditory- and

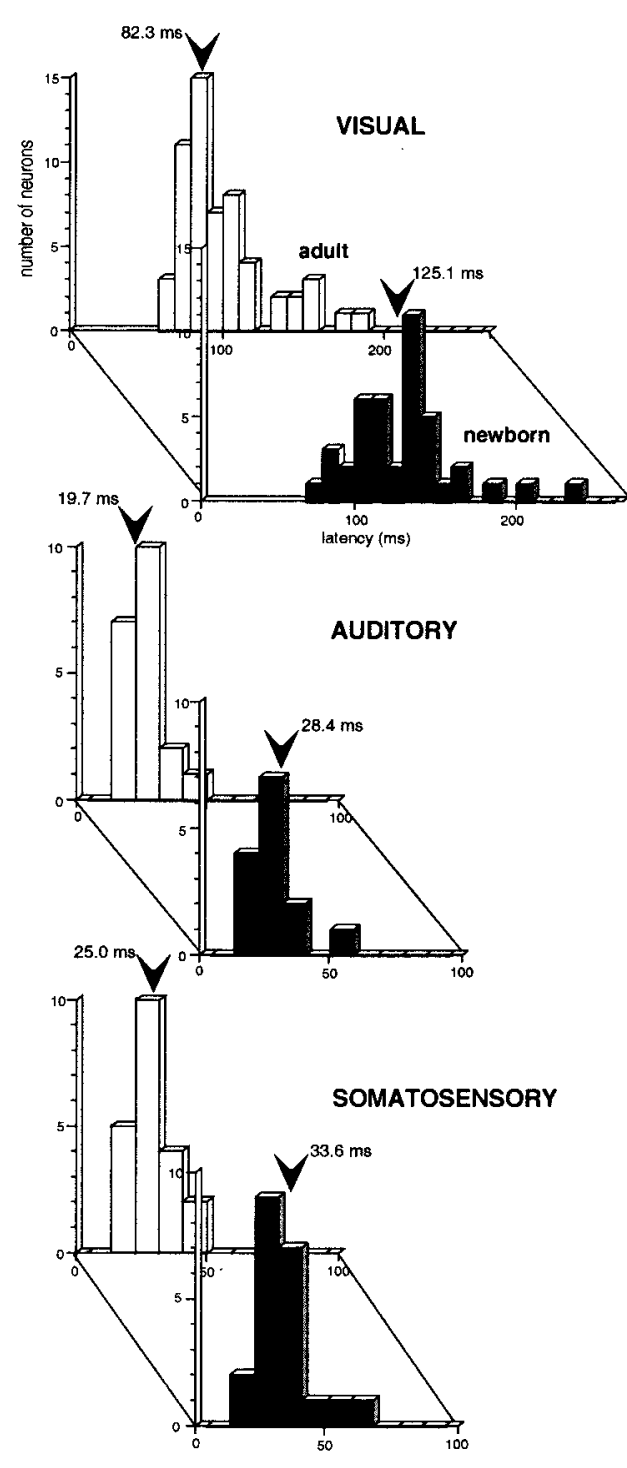

Figure 1. Visual, auditory, and somatosensory latencies are significantly longer in the SC of the newborn (black) than in the SC of the adult (gray). The arrow in each distribution represents the population mean.

somatosensory-responsive neurons tended to be distributed deeper (e.g., lamina VI).

\section{Receptive field size and topography}

Regardless of modality or modality convergence pattern, receptive fields in newborn animals were significantly larger than those in the adult. Representative examples of receptive fields for each neuronal type, as well as plots of receptive field size in newborn and adult animals as a function of SC location, are presented in Figure 3. These data show that visual, auditory, and somatosensory receptive fields were larger in neonates than adults at every SC location sampled. Because there were no systematic differences between the receptive field sizes of modality-specific and multisensory neurons when receptive fields were matched by modality, age, and location, these two neuronal subsets were combined at each age. Highlighting the immaturity of these receptive fields in the newborn, a number of somatosensoryresponsive ( 8 of $29 ; 28 \%$ ) and auditory-responsive ( 3 of $19 ; 16 \%$ ) neurons lacked a traditional receptive field. They responded to stimuli at all locations in contralateral sensory space. 


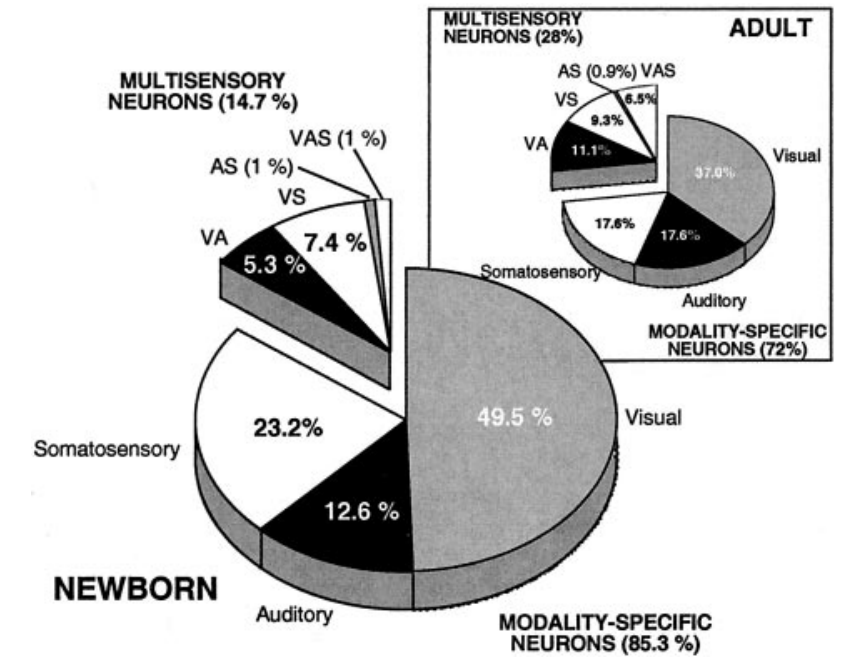

Figure 2. Modality convergence patterns in the SC of the newborn and adult (inset) monkey. Pie charts show the distributions of all recorded sensory-responsive neurons in the multisensory laminas (IV-VII) of the SC.

Despite the immaturity in the size of receptive fields in the newborn, the topographic organization that characterizes the SC representation of visual, auditory, and somatosensory space was already evident at birth. Individual neurons at any given SC site had receptive fields representing similar regions of sensory space. For example, neurons in the rostral SC had receptive fields in central or frontal visual, auditory, and/or somatosensory space, whereas neurons in the caudal SC had peripheral or caudal receptive fields. Neurons in the more medial aspects of the SC had receptive fields in superior sensory space, and those in the more lateral SC had receptive fields in inferior sensory space. This topographic order was particularly evident in the systematic nature of receptive field transitions along electrode penetrations traversing the SC in a rostral-to-caudal oblique track (Fig. 4). The overall topographic register among modalities in the newborn was also reflected in the characteristic cross-modal receptive field register among the different receptive fields of individual multisensory neurons (Fig. 3). Despite the comparatively large receptive fields in the newborn, its within-neuron spatial correspondence approximated that found in adults. Exemplifying this, in the newborn, the mean area of receptive field overlap for visualauditory neurons, depicted as the percentage of the visual receptive field subsumed within the auditory receptive field, was $81 \%$, a value that did not differ significantly from that found in the adult (77\%).

\section{Multisensory integration}

In contrast to the comparatively modest level of immaturity in many of the sensory properties of SC neurons at birth, the immaturity of their multisensory information processing capabilities was striking. Each of the multisensory neurons studied ( $n=$ 14) failed to show the typical response enhancement seen in the adult to spatially and temporally coincident cross-modal stimulus combinations. These response enhancements have been found to be the most consistent index of multisensory integration in both developing and adult animals (Meredith and Stein, 1986a,b; Wallace et al., 1996; Wallace and Stein, 1997). Each of the neurons examined showed the same pattern of results, and 10 of these (four visual-auditory, five visual-somatosensory, and one audi- tory-somatosensory) were maintained long enough to present a full complement of modality-specific and multisensory tests. In each, the initial set of stimuli were chosen to be minimally effective and were presented within the area of receptive field overlap. These stimulus characteristics have been shown to maximize both the probability and magnitude of generating a significantly enhanced multisensory response in adult SC neurons (Meredith and Stein, 1986a,b; Wallace et al., 1996; Kadunce et al., 1997). Nevertheless, in each instance, these cross-modal stimulus combinations failed to evoke a response that was significantly greater than that evoked by the most effective of the modalityspecific component stimuli (Fig. 5). The averaged response enhancements in the populations of newborn and adult SC neurons are shown in Figure 6.

The differing response characteristics (i.e., receptive field size, latency, etc.) of neonatal multisensory SC neurons, coupled with the observation that the temporal constraints on multisensory integration in the SC can be greater in neonatal than adult cats (Wallace and Stein, 1997), suggested that multisensory integration in the newborn monkey might have stimulus requirements that differ from those found at maturity. To examine this possibility, a number of neurons were examined by repeatedly and systematically varying the spatial, temporal, and/or physical characteristics of the cross-modal stimuli presented.

In seven neurons in the newborn, a series of stimulus combinations in which the stimuli were presented at various spatial locations were examined. These combinations included a wide range of stimulus disparities, ranging from coincidence (both stimuli together in space and within the excitatory receptive fields), to significant within-field spatial disparity, to the situation in which one stimulus was presented within its receptive field and the other was presented outside its receptive field. As illustrated in the example shown in Figure $7 A$, in which multiple within-field locations were examined, all such manipulations failed to alter the dynamics of the multisensory response. In each case, the multisensory response continued to closely approximate the response to the most effective modality-specific component stimulus. Furthermore, combinations in which one of the stimuli was positioned outside the receptive field failed to elicit response depression. These findings are in sharp contrast to those in the adult, in which spatially coincident combinations of cross-modal stimuli generally produce significant response enhancement, whereas spatially disparate combinations of cross-modal stimuli often produce response depression (Wallace et al., 1996). In five neurons in the newborn, the cross-modal temporal relationships were also manipulated by varying the SOAs over an interval spanning 500 msec, centered on synchrony. Once again, unlike in the adult, these manipulations failed to substantially change the multisensory responses of neonatal neurons (Fig. 7B).

Last, to examine the possibility that, in contrast to their adult counterparts, neonatal SC neurons would exhibit response enhancements most readily to highly effective rather than weakly effective modality-specific stimuli, the activity of five neurons was examined in response to cross-modal stimulus pairs that were varied substantially in their effectiveness. In each of these neurons, a brief series of pretests was conducted with each of the modality-specific stimuli to which it was responsive. This was done to provide a rough approximation of the dynamic range of the neuron. Although the response range of SC neurons in the neonate was found to be compressed relative to the adult, a reasonable range of responses were still possible in each of these neurons. Thus, the component modality-specific stimulus inten- 


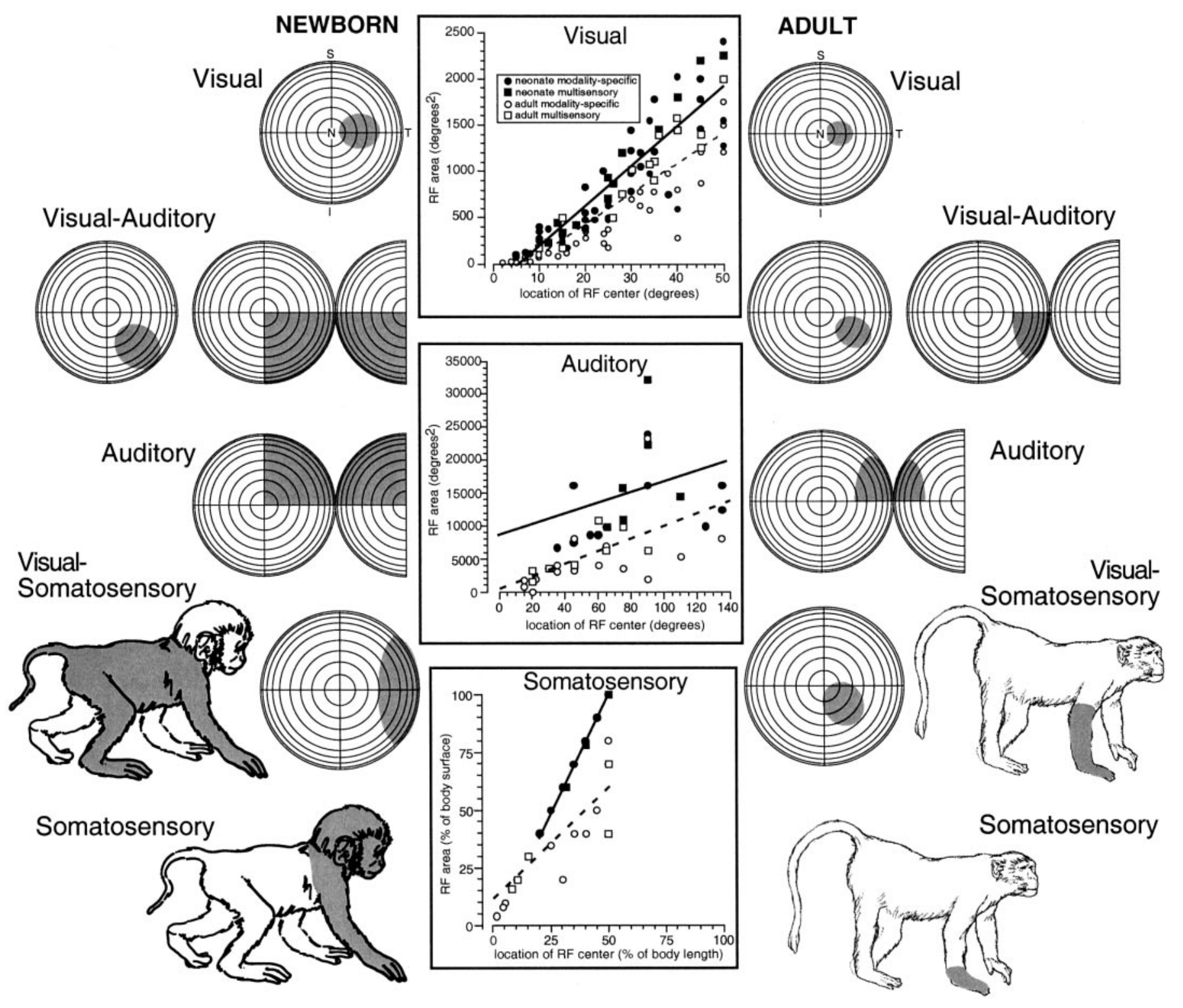

Figure 3. The receptive fields of SC neurons are larger in neonates than in adults. Representative receptive fields (shading) of multisensory and modality-specific neurons, at comparable sites in the multisensory laminas of the newborn (left) and adult (right) SC, are shown on schematics of visual and auditory space and on drawings of the body surface. In the schematics of visual and auditory space, straight lines illustrate the horizontal and vertical meridians, and each concentric circle represents $10^{\circ}$. The half-circle in the auditory schematic represents the caudal half of auditory space. For illustrative purposes, all receptive fields are shown in right (contralateral) sensory space. In the center are plotted the relationships between size and location of modality-specific and multisensory receptive fields. Visual and auditory receptive fields are plotted in areal extent. Somatosensory receptive fields are normalized to represent a percentage of the total body surface, and the somatosensory graph contains only receptive fields whose centers are located on the front half of the body (see Results). Note that, regardless of the modality, modality convergence pattern, or the locations of the receptive field centers of a neuron, neonatal receptive fields (dark symbols and solid lines) are larger than those of their adult counterparts (lighter symbols and dashed lines). $S$, Superior; $I$, inferior; $N$, nasal; $T$, temporal.

sities could be varied, from those that were minimally effective (i.e., threshold) to those that were maximally effective, evoking 5-10 impulses on each stimulus presentation (additional increases in intensity evoked no additional increases in response). The effects of 33 cross-modal stimulus combinations of varying effectiveness were examined in these neurons. In no case did these manipulations yield multisensory response enhancement (Fig. 7C).

\section{DISCUSSION}

The present experiments demonstrate that there are a substantial number of maturationally advanced sensory response properties in SC neurons of the deep (multisensory) laminas in the newborn rhesus monkey. These observations are consistent with previous results showing that its superficial lamina visual neurons also have relatively mature response properties (Wallace et al., 1997) and with the comparative ease and rapidity with which this animal learns to deal with visual, auditory, and somatosensory cues.

Neurons in the deep laminas of the newborn monkey are highly active, already responding to each of the sensory modalities present in the adult, and have modality convergence patterns similar to those found at maturity. Furthermore, the characteristic topographic organization of each of the sensory representa- 


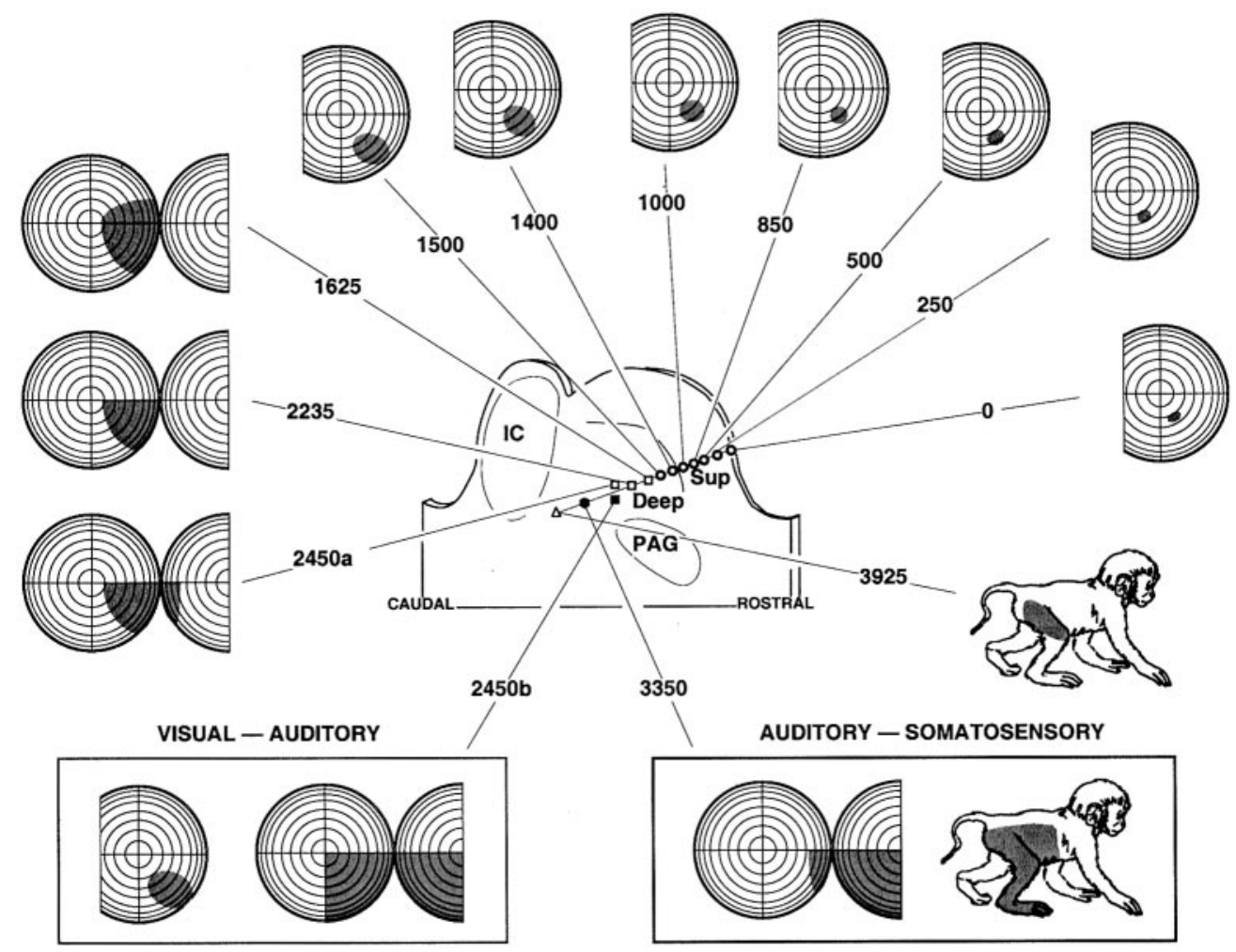

Figure 4. Receptive fields in the newborn's SC shifted systematically as neurons were sampled at progressively more caudal locations. The middle schematic illustrates the path of an electrode on a drawing of a parasagittal section. Each of the symbols represents a sensory-responsive neuron whose receptive field(s) was mapped. Note that, as the electrode advanced, the progression of receptive fields (shading) was from those representing frontal or rostral sensory space to those representing temporal or caudal sensory space. Boxes highlight the receptive fields of multisensory neurons. Numbers represent the recorded depth of each neuron from the surface of the SC. Conventions are the same as in Figure 3. Sup, Superficial layers; Deep, multisensory layers; $P A G$, periaqueductal gray; $I C$, inferior colliculus.
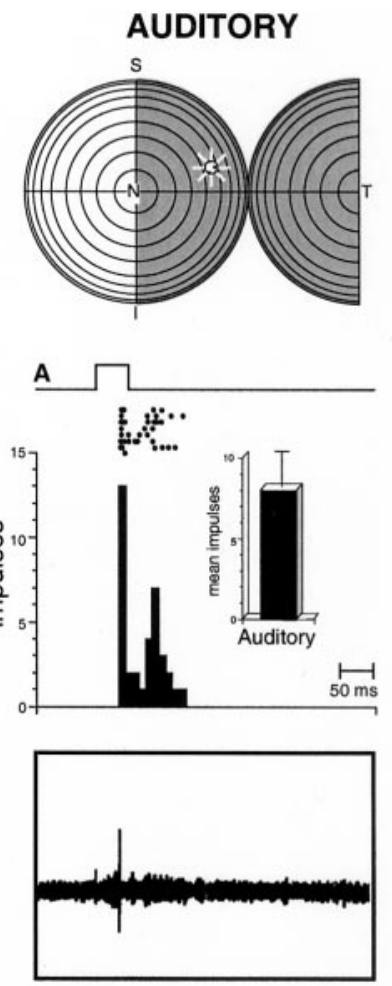

tions in the SC is already evident at birth, and the receptive fields of individual multisensory neurons have levels of cross-modal register that approximate those seen in the adult. Nevertheless, neurons of the deep laminas, like those in the exclusively visual superficial laminas, are not yet fully mature at this time. Response latencies are longer, receptive fields have yet to consolidate to their adult sizes, and the incidence of multisensory neu-

\section{COMBINED}
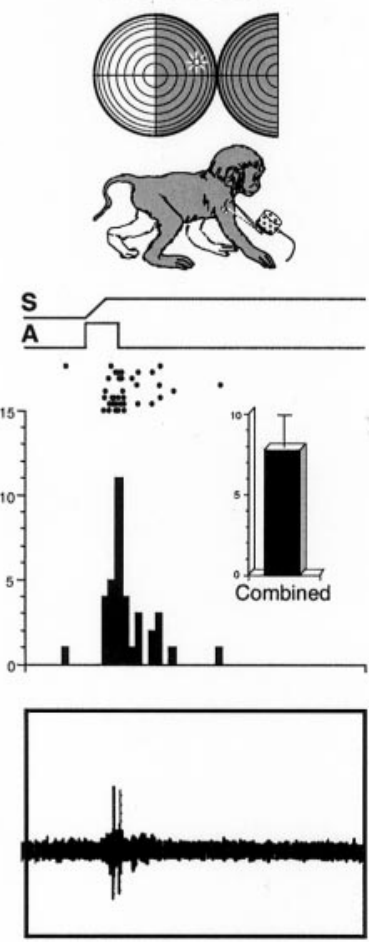

Figure 5. Neurons in the SC of the newborn monkey failed to exhibit mature multisensory integration. Top panels illustrate the auditory and somatosensory receptive fields (shading) of a multisensory neuron in the newborn, as well as the locations of test stimuli (speaker icon and somatosensory probe). Rasters, peristimulus time histograms, summary bar graphs, and oscillographic traces show the responses of this neuron to an auditory stimulus (left), to a somatosensory stimulus (middle), and to their multisensory combination (right). The duration of the auditory $(A$; square wave shows white-noise burst) and somatosensory $(S$; ramp shows probe movement) stimuli are shown. Oscillographic traces at the bottom show the responses of this neuron to a single presentation of each stimulus. Note that the responses to the cross-modal stimulus combination differ little from the responses to the more effective of the two modality-specific stimuli.

rons is approximately half that found later in adulthood (Wallace et al., 1996). Most significant in the current context, however, is the observation that SC neurons do not yet integrate the crossmodal inputs they receive.

In the adult monkey, $83 \%$ of the multisensory neurons in the $\mathrm{SC}$ are capable of synthesizing their cross-modal inputs, as demonstrated by their ability to show significantly enhanced responses 


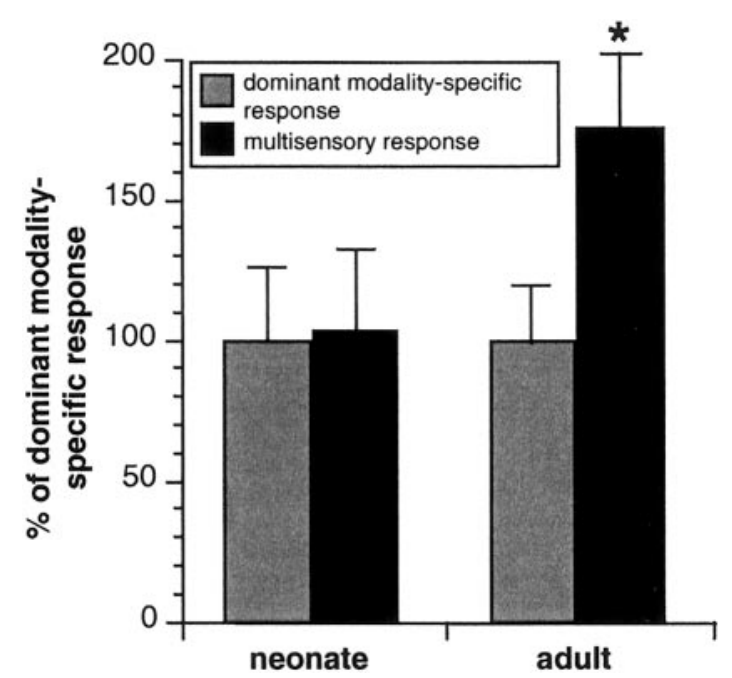

Figure 6. The newborn's multisensory responses were no different from its modality-specific responses. Multisensory SC responses are plotted here as a proportion of the dominant modality-specific response (newborn, left; adult, right). Note that the newborn's multisensory responses differ little from its dominant modality-specific responses (left), whereas these responses differ substantially in the adult (right). ${ }^{*} p<0.01$.

to spatially coincident stimuli (Wallace et al., 1996). However, these same stimuli failed to elicit multisensory enhancements in SC neurons of the newborn. Furthermore, spatially disparate cross-modal stimuli did not elicit multisensory depression. The capability for multisensory integration was not demonstrated in any of the neurons studied here, even after extensive manipulations of the physical characteristics of the modality-specific stimuli and their spatial and temporal relationships. Based on the incidence of neurons exhibiting multisensory integration in the adult, the probability that such a capability was overlooked in the sample of neurons studied in the newborn is $\sim 2 \times 10^{-8}$. However, despite this impressive statistic, the conclusion that the newborn's multisensory SC neurons are incapable of multisensory integration is based on an absence of evidence, an unavoidable problem in maturational studies of this sort. However, lending credence to this conclusion are the similarities to the observations that have been made in the neonatal cat (Wallace and Stein, 1997). In this species, the first multisensory neurons do not appear in the SC until $\sim 10$ postnatal days, and it is not until 3 postnatal weeks that the full complement of modality convergence patterns is evident. Although these early multisensory neurons respond reliably and robustly to sensory stimuli, just as in the newborn monkey, there are few differences between the magnitude of the responses evoked by modality-specific stimuli and those evoked by crossmodal combinations of stimuli. The earliest SC neurons in cat capable of synthesizing cross-modal cues appear at $\sim 4$ postnatal weeks, and their incidence rises continually over the next 3 months.

Based on a number of developmental indices, it appears that the maturational state of the newborn monkey approximates that of the 3-week-old cat (Dreher and Robinson, 1988; Robinson and Dreher, 1990). The absence of multisensory integration at this stage of development in both species appears to be more consistent with the idea that this capacity requires a significant period of postnatal experience than with the idea that it is precluded by the general immaturity of their sensory apparatus or their multisensory SC neurons. Indeed, preliminary observations in cat SC suggest that early visual experience is essential to develop the ability to integrate visual and nonvisual stimuli (Wallace and Stein, 2000; Wallace et al., 2001).

It has been noted previously that, in cat SC, there is a strong correlation between the appearance of multisensory integration and the size of the receptive fields of a multisensory neuron (Wallace and Stein, 1997). Specifically, when the receptive fields of a multisensory neuron have consolidated to $100-150 \%$ of the adult average, its probability of exhibiting multisensory integration is $>50 \%$ (Wallace and Stein, 1997). In the newborn monkey, visual, auditory, and somatosensory receptive fields are, respectively, 163,203 , and $178 \%$ of their adult values. If postparturitional receptive field consolidation takes place at the same rate in monkey and cat and if the index of receptive field size is equally predictive in both species, it is likely that multisensory integration will be seen in at least some monkey SC neurons within the first week of birth. Extrapolating from here, the incidence of integrating multisensory neurons will approximate adult levels within 6-8 weeks of birth. Presumably, it is only at this time that the speeded gaze shifts to cross-modal stimuli will be possible, for these behavioral responses are believed to depend on multisensory integration in SC neurons (Perrott et al., 1990; Hughes et al., 1994; Nozawa et al., 1994; Frens et al., 1995; Goldring et al., 1996; Harrington and Peck, 1998).

Multisensory convergence onto individual neurons is not unique to the SC or to subcortical structures and has been demonstrated in a number of cortical areas in both primate and infraprimate species (Bruce et al., 1981; Rizzolatti et al., 1981; Wallace et al., 1992; Ramachandran et al., 1993; Stein et al., 1993; Fogassi et al., 1996; Graziano et al., 1997, 1999; Duhamel et al., 1998; Schroeder et al., 2001). Most recently, electrophysiological and neuroimaging studies have provided evidence consistent with a similar form of multisensory convergence in human cortex (Giard and Peronnet, 1999; Calvert et al., 2000; Foxe et al., 2000; Bense et al., 2001; Bushara et al., 2001). At least some of these populations of cortical neurons show integrative features that are similar to those found in the SC (Wallace et al., 1992; Ramachandran et al., 1993; Stein et al., 1993). Although it is not yet clear whether the absence of multisensory integration in the neonatal SC reflects an isolated example of structure-specific immaturity, the comparatively early development of the SC lends credence to the idea that, at this developmental stage, there is a general lack of multisensory integration in higher brain centers (i.e., cerebral cortex). If so, the perceptual processes that depend on such multisensory interactions will also be maturationally delayed, a prediction that has implications for studies of sensory development in human infants.

Although a number of studies have attempted to elucidate the multisensory capabilities of the human neonate, little consensus exists as to the timetable for the development of these processes (for review, see Lewkowicz, 2000). Whereas some have posited the newborn to be remarkably multisensory, others have suggested that its sensory modalities function independently and that cross-modal development has a fairly protracted maturational timetable. The present observations, although not directly relevant to perception or to humans, do suggest that any processes that require the kinds of multisensory signal changes described here are unlikely to appear until an individual has had significant postnatal experience with cross-modal cues. Presumably, only then will the enhanced perceptual and behavioral responses (Hughes et al., 1994; Stein et al., 1996; McDonald et al., 2000; 


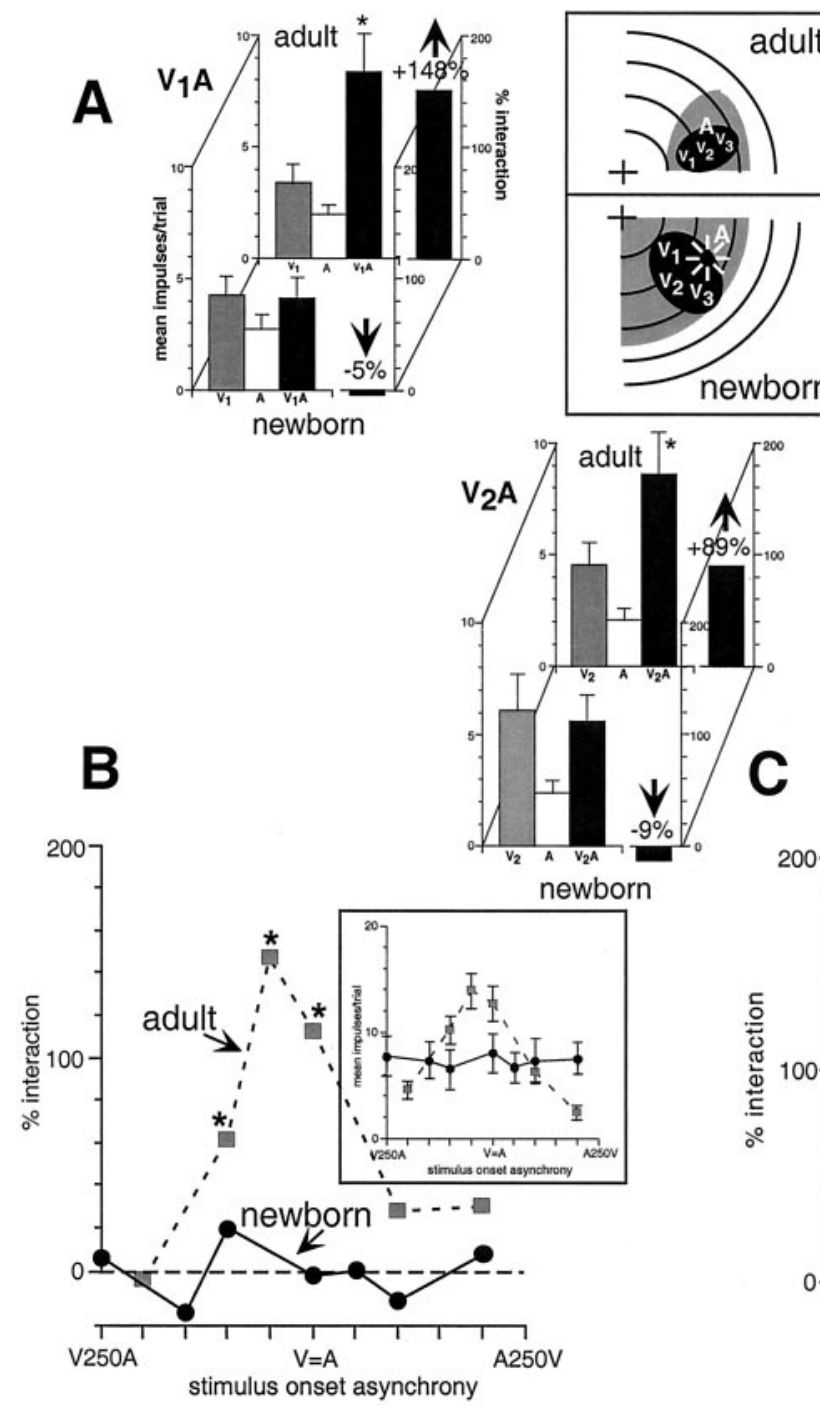

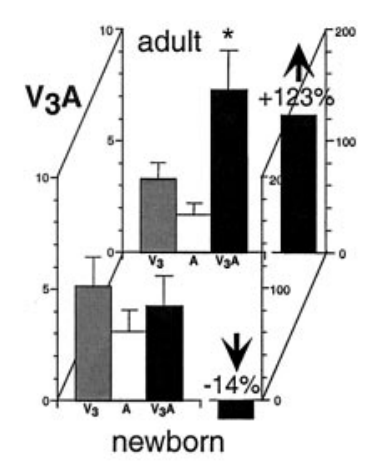

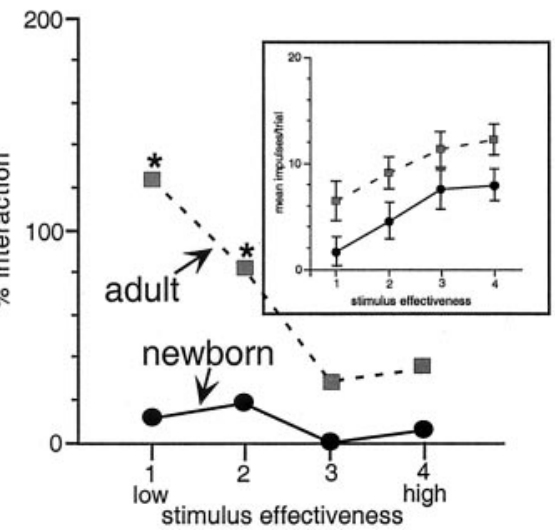

Figure 7. Multisensory response enhancements were not evoked in the newborn's SC, regardless of the spatial or temporal relationship of the crossmodal stimuli or their level of effectiveness. $A$, The visual (darker shading) and auditory (lighter shading) receptive fields of representative multisensory neurons from the newborn and adult are shown in the middle. In these examples, a stationary within-field auditory stimulus $(A)$ was paired with a moving visual stimulus at three different locations within the visual receptive field $(V 1, V 2$, and V3). Summary bar graphs show that, in the newborn, these three crossmodal stimulus pairings $(V 1 A, V 2 A$, and $V 3 A$ ) produced responses that approximated those evoked by the dominant modality-specific stimulus, but in the adult, similar pairings produced substantial multisensory response enhancements. $B$, Multisensory response enhancements peaked at certain cross-modal SOAs in the adult (gray symbols and dashed line), but varying SOA had little impact on the responses of multisensory neurons in the newborn (black symbols and solid line). C, Similarly, multisensory response enhancement in the adult often peaked at low levels of stimulus effectiveness ( gray symbols and dashed line), but manipulations of this stimulus parameter had little impact on the multisensory product in neonatal SC neurons (black symbols and solid line). * $p<0.05$.
Vroomen and de Gelder, 2000) that are believed to depend on this form of multisensory integration be possible.

\section{REFERENCES}

Bense S, Stephan T, Yousry TA, Brandt T, Dieterich M (2001) Multisensory cortical signal increases and decreases during vestibular galvanic stimulation (fMRI). J Neurophysiol 85:886-899.

Boothe RG, Dobson V, Teller DY (1985) Postnatal development of vision in human and nonhuman primates. Annu Rev Neurosci $8: 495-545$.

Bruce C, Desimone R, Gross CG (1981) Visual properties of neurons in a polysensory area in superior temporal sulcus of the macaque. J Neurophysiol 46:369-384.

Bushara KO, Grafman J, Hallett M (2001) Neural correlates of auditoryvisual stimulus onset asynchrony detection. J Neurosci 21:300-304.

Calvert GA, Campbell R, Brammer MJ (2000) Evidence from functional magnetic resonance imaging of crossmodal binding in the human heteromodal cortex. Curr Biol 10:649-657.

Carlson M (1984) Development of tactile discrimination capacity in Macaca mulatta. I. Normal infants. Brain Res 318:69-82.

Dreher B, Robinson SR (1988) Development of the retinof ugal pathway in birds and mammals: evidence for a common "timetable." Brain Behav Evol 31:369-390.

Duhamel JR, Colby CL, Goldberg ME (1998) Ventral intraparietal area of the macaque: congruent visual and somatic response properties. J Neurophysiol 79:126-136.

Fogassi L, Gallese V, Fadiga L, Luppino G, Matelli M, Rizzolatti G (1996) Coding of peripersonal space in inferior premotor cortex (area F4). J Neurophysiol 76:141-157.

Foxe JJ, Morocz IA, Murray MM, Higgins BA, Javitt DC, Schroeder CE (2000) Multisensory auditory-somatosensory interactions in early cor- tical processing revealed by high-density electrical mapping. Brain Res Cogn Brain Res 10:77-83.

Frens MA, Van Opstal AJ, Van der Willigen RF (1995) Spatial and temporal factors determine auditory-visual interactions in human saccadic eye movements. Percept Psychophys 57:802-816.

Giard MH, Peronnet F (1999) Auditory-visual integration during multimodal object recognition in humans: a behavioral and electrophysiological study. J Cognit Neurosci 11:473-490.

Goldring JE, Dorris MC, Corneil BD, Ballantyne PA, Munoz DP (1996) Combined eye-head gaze shifts to visual and auditory targets in humans. Exp Brain Res 111:68-78.

Graziano MS, Hu XT, Gross CG (1997) Visuospatial properties of ventral premotor cortex. J Neurophysiol 77:2268-2292.

Graziano MS, Reiss LA, Gross CG (1999) A neuronal representation of the location of nearby sounds. Nature 397:428-430.

Harrington LK, Peck CK (1998) Spatial disparity affects visual-auditory interactions in human sensorimotor processing. Exp Brain Res 122:247-252.

Hughes HC, Reuter-Lorenz PA, Nozawa G, Fendrich R (1994) Visualauditory interactions in sensorimotor processing: saccades versus manual responses. J Exp Psychol Hum Percept Perform 20:131-153.

Kadunce DC, Vaughan JW, Wallace MT, Benedek G, Stein BE (1997) Mechanisms of within- and cross-modality suppression in the superior colliculus. J Neurophysiol 78:2834-2847.

Kadunce DC, Vaughan JW, Wallace MT, Stein BE (2001) The influence of visual and auditory receptive field organization on multisensory integration in the superior colliculus. Exp Brain Res 139:303-310.

Kao CQ, McHaffie JG, Meredith MA, Stein BE (1994) Functional development of a central visual map in cat. J Neurophysiol 72:266-272.

King JE, Fobes JT, Fobes JL (1974) Development of early behaviors in neonatal squirrel monkeys and cotton-top tamarins. Dev Psychobiol 7:97-109. 
Kiorpes L, Movshon JA (1998) Peripheral and central factors limiting the development of contrast sensitivity in macaque monkeys. Vision Res 38:61-70.

Lewkowicz DJ (2000) The development of intersensory temporal perception: an epigenetic systems/limitations view. Psychol Bull 126:281-308.

McDonald JJ, Teder-Salejarvi WA, Hillyard SA (2000) Involuntary orienting to sound improves visual perception. Nature 407:906-908.

McHaffie JG, Stein BE (1983) A chronic headholder minimizing facial obstructions. Brain Res Bull 10:859-860.

Meredith MA, Stein BE (1986a) Spatial factors determine the activity of multisensory neurons in cat superior colliculus. Brain Res 365:350-354.

Meredith MA, Stein BE (1986b) Visual, auditory, and somatosensory convergence on cells in superior colliculus results in multisensory integration. J Neurophysiol 56:640-662.

Meredith MA, Stein BE (1996) Spatial determinants of multisensory integration in cat superior colliculus neurons. J Neurophysiol 75:1843-1857.

Meredith MA, Nemitz JW, Stein BE (1987) Determinants of multisensory integration in superior colliculus neurons. I. Temporal factors. J Neurosci 7:3215-3229.

Norton TT (1974) Receptive-field properties of superior colliculus cells and development of visual behavior in kittens. J Neurophysiol 37:674-690.

Nozawa G, Reuter-Lorenz PA, Hughes HC (1994) Parallel and serial processes in the human oculomotor system: bimodal integration and express saccades. Biological Cybernetics 72:19-34.

Perrott DR, Saberi K, Brown K, Strybel TZ (1990) Auditory psychomotor coordination and visual search performance. Percept Psychophys 48:214-226.

Ramachandran R, Wallace MT, Clemo HR, Stein BE (1993) Multisensory convergence and integration in rat cortex. Soc Neurosci Abstr 19:1447.

Rizzolatti G, Scandolara C, Matelli M, Gentilucci M (1981) Afferent properties of periarcuate neurons in macaque monkeys. II. Visual responses. Behav Brain Res 2:147-163.

Robinson SR, Dreher B (1990) The visual pathways of eutherian mammals and marsupials develop according to a common timetable. Brain Behav Evol 36:177-195.

Schroeder CE, Lindsley RW, Specht C, Marcovici A, Smiley JF, Javitt DC (2001) Somatosensory input to auditory association cortex in the macaque monkey. J Neurophysiol 85:1322-1327.

Stein BE, Gallagher HL (1981) Maturation of cortical control over superior colliculus cells in cat. Brain Res 223:429-435.
Stein BE, Meredith MA (1993) The merging of the senses. Cambridge, MA: MIT.

Stein BE, Labos E, Kruger L (1973a) Sequence of changes in properties of neurons of superior colliculus of the kitten during maturation. J Neurophysiol 36:667-679.

Stein BE, Labos E, Kruger L (1973b) Determinants of response latency in neurons of superior colliculus in kittens. J Neurophysiol 36:680-689.

Stein BE, Clamann HP, Goldberg SJ (1980) Superior colliculus: control of eye movements in neonatal kittens. Science 210:78-80.

Stein BE, Meredith MA, Huneycutt W, McDade L (1989) Behavioral indices of multisensory integration: orientation to visual cues is affected by auditory stimuli. J Cognit Neurosci 1:12-24.

Stein BE, Meredith MA, Wallace MT (1993) The visually responsive neuron and beyond: multisensory integration in cat and monkey. Prog Brain Res 95:79-90.

Stein BE, London N, Wilkinson LK, Price DD (1996) Enhancement of perceived visual intensity by auditory stimuli: a psychophysical analysis. J Cognit Neurosci 8:497-506.

Vroomen J, de Gelder B (2000) Sound enhances visual perception: cross-modal effects of auditory organization on vision. J Exp Psychol Hum Percept Perform 26:1583-1590.

Wallace MT, Stein BE (1994) Cross-modal synthesis in the midbrain depends on input from cortex. J Neurophysiol 71:429-432.

Wallace MT, Stein BE (1997) Development of multisensory neurons and multisensory integration in cat superior colliculus. J Neurosci 17:2429-2444.

Wallace MT, Stein BE (2000) The role of experience in the development of multisensory integration. Soc Neurosci Abstr 26:1220.

Wallace MT, Meredith MA, Stein BE (1992) Integration of multiple sensory modalities in cat cortex. Exp Brain Res 91:484-488.

Wallace MT, Meredith MA, Stein BE (1993) Converging influences from visual, auditory, and somatosensory cortices onto output neurons of the superior colliculus. J Neurophysiol 69:1797-1809.

Wallace MT, McHaffie JG, Stein BE (1995) Sensory response properties in the superior colliculus (SC) of the newborn rhesus monkey. Soc Neurosci Abstr 21:655.

Wallace MT, Wilkinson LK, Stein BE (1996) Representation and integration of multiple sensory inputs in primate superior colliculus. J Neurophysiol 76:1246-1266.

Wallace MT, McHaffie JG, Stein BE (1997) Visual response properties and visuotopic representation in the newborn monkey superior colliculus. J Neurophysiol 78:2732-2741.

Wallace MT, Hairston WD, Stein BE (2001) Long-term effects of darkrearing on multisensory processing. Soc Neurosci Abstr, in press. 